\title{
Self-Reported Adherence to the Use of Clinical Practice Guidelines of Hypertensive Disorders of Pregnancy in Jordanian Hospitals
}

\author{
Sarah M. Alja'freh ${ }^{1}$, Lubna Abu-Shaikha ${ }^{2}$ \\ ${ }^{1}$ RN, MSN, PhD, Full-time lecturer, Department of Medical Sciences Al-Balqa'Applied University, Salt 19117. \\ Jordan, ${ }^{2} R N$, MSN, PhD, Professor, Maternal \& Child Health Nursing, School of Nursing, the University of Jordan
}

\begin{abstract}
Background: Hypertensive Disorders of Pregnancy (HDP) represents a life-threatening event for women and their families. A number of national and international Clinical Practice Guidelines (CPGs) of HDP have been published, however their use in clinical practice is still not well documented as it is complex. Suboptimal adherence to CPGs may lead to unfavorable maternal and perinatal outcomes. The purpose of this study is to assess the level of adherence of Healthcare Providers (HCPs) including obstetricians, nurses, and midwives to the HDP CPG based on Health System Strengthening (HSS II) recommendations in Jordan.

Methods: A cross-sectional study design is used. 270 HCPs are recruited from different healthcare sectors that provide maternal health care services to women who are at risk of developing HDP. Data is collected through self-reported questionnaire.

Conclusion: HCPs showed a suboptimal adherence of to evidence-based recommendations of HDP's guidelines; particularly in the antepartum and postpartum period. The results of this study can be used by guideline developers to raise acceptance and applicability of the guideline recommendations. Also, it may help healthcare stakeholders and policy makers in designing tailored strategies to ensure successful and sustainable guideline adoption and its implementation in evidence-based maternity care.
\end{abstract}

Keywords: Clinical Practice Guidelines, Hypertensive Disorders of Pregnancy, Jordanian Healthcare Sector, Maternal Healthcare, Self-reported Adherence,

\section{Introduction}

Globally, about 830 women and 7,700 newborn die every day due to largely preventable complications that occur at any time during pregnancy, childbirth and postpartum periods ${ }^{1}$. The most common leading causes of preventable Severe Acute Maternal Morbidity (SAMM) cases were HDP (particularly; pre-eclampsia and eclampsia), hemorrhage/blood loss, and septicemia 2, 3. Globally, HDP affects approximately $8-10 \%$ of all pregnancies ${ }^{4-6}$. It is the second direct cause of maternal deaths representing around $16 \%$ of the estimated global maternal deaths ${ }^{7}$.
In Jordan, HSS II is USAID-funded project that work jointly with different healthcare sectors to improve access and quality of maternal healthcare services. One of its major purposes is to support the development of clinical practice guidelines, nursing procedures, and service standards for maternal, neonatal, and family planning services. Since 2009, The HSS II CPGs of HDP have been published till this time. The HSS II guideline of HDP comprised of a set of evidence based recommendations. Obstetricians, nurses, and midwives are key providers in assessing, diagnosing, and managing high-risk pregnant women through the use of the best available evidence in their clinical practice to improve maternal and neonatal health care. HCPs' adherence to 
CPGs and protocols will lessen improper variations of clinical practice, synthesize the best available evidence, and ensure the provision of high-quality evidencebased care. To our best knowledge there have been no published studies in Jordan regarding self-reported adherence to the use of CPGs of HDP.

\section{Material and Methods}

\section{Study Design, Study Instrument and Sampling technique}

A cross-sectional study design was used. The study sites included four hospitals: two governmental, one military, and one private, thus representing the main healthcare sectors in Jordan. This study was conducted during December 2017 to March 2018. A questionnaire was developed by the first author. The data were collected using this self-reported questionnaire that composed of two sections: the first section consisted of demographic and professional characteristics, and the second section composed of a number of evidence-based recommendations that were adopted from the HDP's CPG of the HSS II (2011). The questionnaire was pilot-tested among a group of 30 maternal healthcare providers. Volunteer sampling technique was used to recruit the participants. A cover letter explaining the nature and purpose of the study was attached with the questionnaire.

\section{Inclusion Criteria}

The eligible HCPs for the study were obstetricians, nurses, and midwives who agreed to participate in the study, provide bedside care for pregnant women who are at risk of developing or experience HDP in their antepartum, intrapartum, and postnatal period, and have at least two years of clinical experience in Obstetrical and Gynecological Units. The study was approved by the selected Hospital and the ethical committee at the University of Jordan.

\section{Data Management and Statistical Analysis}

Healthcare providers were asked to report whether they are adherent to each statement as $0 \%$ of the time "Never", 25\% of the time "Rarely", $50 \%$ of the time "Sometimes", $75 \%$ of the time "Often", or 76\%$100 \%$ of the time "Almost Always". Those who report implementing CPGs $76 \%-100 \%$ of the time ("Almost Always") were classified as "adherent", while others were classified as "non-adherent". The principal researcher collected the completed questionnaires in sealed envelopes to maintain confidentiality and anonymity of the participants.

Collected Data were entered by the researcher to the SPSS (version 21) spreadsheet and coded in accordance with the variables' level of measurement. The data were rechecked for consistency by the other author and cleaned. Descriptive statistics were used to summarize the characteristics of the study population. Mean and standard deviation were used to summarize continuous variables, and frequency and percentages were used to summarize categorical variables. For the analysis, the categorical variables were compared using Pearson chisquared test or Fisher's exact test. P-value below 0.05 was considered statistically significant.

\section{Ethical Considerations}

Ethical approval was obtained from the ethical committee at University of Jordan. Also the permission has been obtained from the authority at each selected hospitals for conducting the research study. All of the participants verbally consented, agreed willingly and voluntarily to participants in the study.

\section{Results}

The researcher approached 348 eligible participants who provide care for pregnant women. The filled questionnaires were returned by 270 (77.6\% response rate) of HCPs. 
Table 1 Socio-demographic and Professional Characteristics of the HCPs $(n=270)$

\begin{tabular}{|c|c|c|}
\hline Socio-demographic Variables & Mean & Standard Deviation \\
\hline Age (years) & 31.0 & 6.0 \\
Clinical Experience (years) & 8.25 & 5.6 \\
\hline Professional Characteristics & Frequency & Percent (\%) \\
\hline Professional Discipline & & \\
Obstetrician & 58 & $21.5 \%$ \\
Staff Nurse & 36 & $13.3 \%$ \\
Registered Midwife & 158 & $58.5 \%$ \\
Nurse-Midwife & 18 & $6.7 \%$ \\
\hline Sector of Employment & & $58.9 \%$ \\
Governmental & 159 & $31.1 \%$ \\
Royal medical Services & 84 & $10.0 \%$ \\
\hline Private & 27 & \\
\hline
\end{tabular}

HCP: Healthcare Providers

Nurses' and Midwives' Self-reported Adherence to the HSS II Guideline of HDP

The "HSS II guideline of HDP" consists of three sections of evidence-based recommendations regarding the care provided by nurses and midwives for women with HDP throughout the antepartum, intrapartum, and postpartum periods. According to their area of clinical practice, $48.1 \%(\mathrm{n}=102)$ of nurses and midwives responded to the antepartum recommendations, 34.9\% $(\mathrm{n}=74)$ responded to the intrapartum recommendations, and $17.0 \% \quad(n=36)$ responded to the postpartum recommendations of the guideline. Overall, the selfreported adherence rate of nurses and midwives across all evidence-based recommendations of the HSS II guideline of HDP in the antepartum, intrapartum, and postpartum sections was $18.6 \%(n=19), 59.5 \%(n=44)$, and $33.3 \%(n=12)$, respectively.
Obstetricians' Self-reported Adherence to the Health System Strengthening (HSS II) Guideline of Hypertensive Disorders of Pregnancy

The HSS II guideline of HDP consists of three sections of evidence-based recommendations regarding the care provided by obstetricians for women with HDP throughout the antepartum, intrapartum, and postpartum periods. According to their clinical area of practice, $41.4 \%(n=24)$ of obstetricians responded to the antenatal part, $36.2 \%(n=21)$ responded to the intrapartum part, and $22.4 \%(n=13)$ responded to the postnatal part of the guidelines. Overall, the self-reported adherence rate to evidence-based recommendations of the HSS II guideline of HDP in the antepartum, intrapartum, and postpartum sections was $(45.8 \%, \mathrm{n}=11),(71.4 \%, \mathrm{n}=15)$, and $(30.8 \%, n=4)$, respectively (table 3 ) 
Table 3: Obstetricians' Self-reported Adherence to the HSS II Guideline of HDP

\begin{tabular}{|c|c|c|}
\hline Recommendations of HSS II Guideline of HDP for Obstetricians & $\begin{array}{l}\text { Adherent } \\
\text { n (\%) }\end{array}$ & $\begin{array}{l}\text { Non Adherent } \\
\text { n (\%) }\end{array}$ \\
\hline Overall self-reported adherence rate to antepartum recommendations $($ Total $=24)$ & $\begin{array}{l}45.8 \% \\
(n=11)\end{array}$ & $\begin{array}{l}54.2 \% \\
(n=13)\end{array}$ \\
\hline Calcium supplementation during pregnancy in areas where calcium intake is low & $2(8.3)$ & $22(91.7)$ \\
\hline $\begin{array}{l}\text { Low-dose acetylsalicylic acid (aspirin, } 75 \mathrm{mg} \text { ) for the prevention of pre- eclampsia in } \\
\text { women at high risk of developing the condition }\end{array}$ & $5(20.8)$ & 19(79.2) \\
\hline Antihypertensive drugs for pregnant women with severe hypertension & $18(75.0)$ & $6(25.0)$ \\
\hline $\begin{array}{l}\text { If there is a viable fetus and the pregnancy is less than } 36 \text { (plus } 6 \text { days) weeks of } \\
\text { gestation, expectant management should be considered in women with severe pre- } \\
\text { eclampsia }\end{array}$ & $9(37.5)$ & $15(62.5)$ \\
\hline Intrapartum Recommendations $($ Total $=21)$ & $\begin{array}{l}71.4 \% \\
(n=15)\end{array}$ & $\begin{array}{l}28.6 \\
(n=6)\end{array}$ \\
\hline $\begin{array}{l}\text { Induction of labor for women with severe pre-eclampsia at a gestational age when the } \\
\text { fetus is not viable or is unlikely to achieve viability within one or two weeks }\end{array}$ & $5(23.8)$ & $16(76.2)$ \\
\hline Expedited delivery for women with severe pre-eclampsia at term & $16(76.2)$ & $5(23.8)$ \\
\hline $\begin{array}{l}\text { Magnesium sulfate, in preference to other anticonvulsants, for the prevention of } \\
\text { eclampsia in women with severe pre-eclampsia }\end{array}$ & $9(42.9)$ & $12(57.1)$ \\
\hline $\begin{array}{l}\text { The full intravenous or intramuscular magnesium sulfate regimen for the prevention } \\
\text { and treatment of eclampsia }\end{array}$ & $9(42.9)$ & $12(57.1)$ \\
\hline $\begin{array}{l}\text { For women with severe pre-eclampsia or eclampsia, use the magnesium sulfate } \\
\text { loading dose followed by immediate transfer to a higher-level health care facility }\end{array}$ & $10(47.6)$ & $11(52.4)$ \\
\hline Intrapartum Recommendations (Total $=13$ ) & $\begin{array}{c}30.8 \% \\
(n=4)\end{array}$ & $\begin{array}{c}69.2 \% \\
(\mathrm{n}=9)\end{array}$ \\
\hline $\begin{array}{l}\text { Continued antihypertensive drugs during the postpartum period for women treated } \\
\text { with antihypertensive drugs during the antenatal period care }\end{array}$ & $3(23.1)$ & 10(76.9) \\
\hline Antihypertensive drugs for women with severe postpartum hypertension & $6(46.2)$ & $7(53.8)$ \\
\hline
\end{tabular}

HSS: Health System Strengthening, HDP: Hypertensive Disorders of Pregnancy

\section{Discussion}

This is the first study that assesses the level of adherence to CPGs of HDP amongst HCPs (including obstetricians, midwives, and nurses) in their clinical practice in Jordanian hospitals.

Nurses and midwives are the first healthcare personnel who make contact with pregnant women with high-risk conditions. Therefore, it is of the utmost 
importance to provide care which is guided by the best available scientific evidence. In the context of maternity care, providers' optimal adherence to clinical guidelines in their daily practice was found to reduce the risks of maternal and neonatal complications (e.g., preterm delivery, low birth weight, stillbirths) particularly in high risk pregnancies ${ }^{8}$.

Results of this study have indicated a low adherence rate among nurses and midwives to a number of recommendations of HDP's clinical guideline. Low adherence rate is congruent with findings from previous studies in Jordan which reported that a number of maternal clinical practices in Jordanian hospitals have been found to be inconsistent with evidence-based recommendations and guidelines ${ }^{9-12}$. Furthermore, similar results were reported by published reports from HSS II project with the MOH in Jordan which indicated a lack of standardized evidence-based guidelines and clinical performance checklists to monitor and manage women who experience, or are at risk for developing Preeclampsia, Eclampsia, and other hypertensive disorders.

In this study, a significant proportion of nurses and midwives reported lack of adherence to the recommendation regarding "screening for clinical risk markers including multifetal pregnancy (46.1\%, $\mathrm{n}=47)$, obesity $(41.2 \%, \mathrm{n}=42)$, and advanced maternal age $(31.4 \%, n=32) "$. This is worthwhile, since current evidence shows that either alone or in combination identified clinical risk markers such as multiple pregnancy, maternal age $>40$ years, pre-pregnancy body mass index $(\mathrm{BMI})>30 \mathrm{~kg} / \mathrm{m}^{2}$ were associated with a significantly heightened risk of Pre-eclampsia ${ }^{13,14}$. This finding suggests strengthening healthcare providers' surveillance and clinical assessment capabilities to improve the pregnancy outcomes for the mother and her fetus.

Upon admission to the labor room, low adherence rate was reported by nurses and midwives in regards to some basic elements of general and obstetrical physical examination such as monitoring of blood pressure every 15 minutes, examination of lower limbs for edema, and checking fundal height. This is against evidence-based recommendations of careful maternal monitoring for blood pressure on a regular basis (at least 4 hourly), signs of deteriorating mother's health condition, and signs of placental insufficiency and growth restriction ${ }^{14}$.

Furthermore, for women who were diagnosed with severe Pre-eclampsia or impending Eclampsia, Magnesium sulfate (MgSO4) is the preferable drug of choice. It has been documented to be associated with a significant reduction in the risk of seizures and maternal mortality, and could have some potential benefits to the unborn baby (e.g., good APGAR score, short hospitalization). Administration of $\mathrm{MgSO} 4$ requires close monitoring of blood pressure, observation of respiratory rate, urine output, and oxygen saturation, and assessing deep tendon reflexes to avoid toxicity ${ }^{14}$. However, nurses and midwives in this study reported lack of adherence to this kind of recommendations. This is undesirable, as evidence suggests that magnesium toxicity is monitored by appropriate clinical monitoring not certainly magnesium serum levels ${ }^{15}$.

Suboptimal adherence to evidence-based recommendation of routine screening and monitoring may reflect lack of knowledge among nurses and midwives about the pathophysiology of the disease and their active role in monitoring the progress of mother's condition and appropriate management in order to decrease the risk of any complications and so, improve maternal and neonatal outcomes. Comparable results were yielded by a number of previous studies which evaluated adherence to standard care and existing guidelines for Hypertensive Disorders of Pregnancy in different countries, in which recommendations for daily monitoring and charting (e.g., blood pressure, deep tendon reflexes, vital signs, level of consciousness (LoC), fetal heart rate and input and output), and monitoring of patients on magnesium sulfate were reported to be the least adherence ${ }^{16-19}$.

Tucker et al (2003) reported in a study on 117 Scottish midwives who provided a management plan of mild non-proteinuria hypertension in pregnancy, only six midwives (5\%) correctly described all the parts of the 
basic surveillance according to the existing guideline. In regards to the elements of the basic surveillance, only ten midwives $(9 \%)$ reported they would check blood pressure twice weekly, urine twice weekly, 13 (11\%) would check blood pressure and urine more than twice weekly, and only one or two reported checking maternal and fetal wellbeing or blood tests ${ }^{20}$.

Commonly many women with Chronic Hypertension during pregnancy are at increased risk of persistent or exacerbated hypertension in the postpartum period. Evidence indicates that measurement of woman's blood pressure should be considered as an integral part of the complete postnatal examination. In the first seven to ten days after delivery, woman's blood pressure should be measured at least every other day, and then weekly for the six weeks of the postpartum period ${ }^{14-20}$. Unfortunately, recommendations regarding "continuous surveillance of blood pressure 72 hours after delivery and again 7 to 10 days later if symptoms persist" were less adhered to in this study. This indicates the necessity of educating nurses and midwives about the continued monitoring and reporting of unremitting symptoms in the postpartum period to minimize any possible complications.

Conversely, obstetricians in this study showed relatively good adherence rate to most of evidence based recommendations of HDP's guideline in regards to prescription of antihypertensive medications, expectant management in women with severe Pre-eclampsia if there is a viable fetus and the pregnancy is less than 36 (plus 6 days) weeks of gestation, induction of labor or expedited delivery for women with severe Preeclampsia, Magnesium sulfate regimen, and continued prescription of antihypertensive medications for women with persistent hypertension in the postpartum period.

Despite the strong recommendation of WHO regarding the efficacy of low-dose acetylsalicylic acid (aspirin $75 \mathrm{mg}$ ) and calcium supplementation for the prevention of Pre-eclampsia in areas with low dietary intake and a high risk of Pre-eclampsia, obstetricians in this study reported low adherence to both recommendations which indicates substandard care and a significant variation in clinical practice of obstetricians which may lead to unfavorable maternal and neonatal outcomes. This finding is against recent evidence that advocate for the administration of low-dose aspirin from 12 weeks' gestation until delivery for high-risk women to reduce the risk of developing Pre-eclampsia by up to $17-50 \%$ and lessen associated fetal and maternal adverse outcomes ${ }^{22,1,23}$.

Some limitations of this study need to be mentioned. Healthcare providers' adherence was measured solely by self-report method. This method may not reflect actual practice; so adherence may be overestimated due to professional expectations and social desirability bias. To minimize this potential bias, anonymity and confidentiality of participants' responses were assured. Despite a high response rate (78\%), study participants were recruited using volunteer sampling which may have introduced selection bias.

\section{Recommendations}

Findings of this study suggest a need for greater efforts in promoting evidence-based practice of nurses and midwives in caring for women who experience HDP throughout antepartum, labor, and postpartum period. Since HDP can occur without warning or with the gradual development of symptoms and proven methods to prevent the disease are nonexistent, early recognition of the disease to optimize maternal and perinatal outcomes is a key goal.

Throughout the labor and postpartum period, the study findings suggest potential areas for further improvement in the care provided by nurses and midwives for women with HDP including complete general and obstetrical physical examination at hospital admission, monitoring of maternal and fetal status as labor progresses, and continuous surveillance of blood pressure measurements. Also, nurses and midwives can do much in the administrative role. Nursing managers should develop and supervise the implementation of clinical guidelines to ensure that nurses and midwives understand and follow these guidelines and its' evidence based recommendations and to periodically assess the 
effectiveness of such guidelines whenever possible. Further attention should be given to provide sufficient resources to meet educational needs and strategies to motivate healthcare providers and promote selfconfidence in relation to clinical guidelines. In addition, greater focus should be placed on continuous monitoring of professional conduct in regards to implementation of HDP's clinical guideline in different healthcare settings.

Future studies are required with more advanced tools and methods (such as qualitative, observational) from the perspectives of key stakeholders, policy makers and patients to better understand the relationship between healthcare providers' perceived barriers to guideline's implementation and their level of adherence to these guidelines.

Findings of the current study suggested that clinical practice guideline, its evidence-based recommendations, and its importance in optimizing maternal and perinatal outcomes should be integrated in nursing and midwifery educational curricula in Jordanian universities and community colleges. Nursing and midwifery students should be educated about the potential benefits of clinical practice guidelines for patients, healthcare professionals, and healthcare system in the context of maternal care. Also, they should be trained and motivated to use clinical guidelines as a familiar part of their practice to provide a more consistent and efficient patient care.

Acknowledgements: This study was supported by University of Jordan. The authors would like to express their gratitude to the healthcare providers who participated in the study and to the staff of the selected hospitals.

Conflicts of Interest: the authors declare no conflict

Funding: None

Ethical Clearance: The study was approved by the scientific research committee at the University of Jordan (2017-2018).

\section{References}

1. Works Health Organization. Maternal mortality: key facts. Geneva; 2019.

2. Lawton B, MacDonald EJ, Brown SA, Wilson L, Stanley J, Tait JD, Dinsdale RA, Coles CL, Geller SE. Preventability of severe acute maternal morbidity. AJOG. 2014 Jun 1;210(6):557-e1.

3. Ghazal-Aswad S, Badrinath P, Sidky I, Safi TH, Gargash H, Abdul-Razak Y, Mirghani H. Severe acute maternal morbidity in a high-income developing multiethnic country. Matern child health j. 2013 Apr 1;17(3):399-404.

4. Yelikar KA, Deshpande SS, Deshmukh SF. Severe Acute Maternal Morbidity in a Tertiary Care Centre with Basic Intermediate Respiratory Care Units Setup. Int. J, of Sci. study. 2015;5(3):36-40.

5. Duley L. The global impact of pre-eclampsia and eclampsia. Semin. Perinatol. 2009 Jun 1 (Vol. 33, No. 3, pp. 130-137). WB Saunders.

6. Jim B, Sharma S, Kebede T, Acharya A. Hypertension in pregnancy: a comprehensive update. Cardiol. Rev. 2010 Jul 1;18(4):178-89.

7. Say L, Chou D, Gemmill A, Tunçalp Ö, Moller AB, Daniels J, Gülmezoglu AM, Temmerman M, Alkema L. Global causes of maternal death: a WHO systematic analysis. Lancet Glob health. 2014 Jun 1;2(6):e323-33.

8. Amoakoh-Coleman M, Klipstein-Grobusch K, Agyepong IA, Kayode GA, Grobbee DE, Ansah EK. Provider adherence to first antenatal care guidelines and risk of pregnancy complications in public sector facilities: a Ghanaian cohort study. BMC Pregnancy Childbirth. 2016 Dec;16(1):1-0.

9. Shaban IA, Hatamleh R, Khresheh R, Homer C. Childbirth practices in Jordanian public hospitals: consistency with evidence-based maternity care? Int. J. EVID-BASED HEA. 2011 Mar;9(1):25-31.

10. Mohammad KI, Shaban I, Homer C, Creedy D. Womens satisfaction with hospital-based intrapartum care: a Jordanian study. IJNM. 2014 Jul 31;6(3):32-9.

11. Khresheh R, Homer C, Barclay L. A comparison of labour and birth outcomes in Jordan with WHO guidelines: a descriptive study using a new birth record. Midwifery. 2009 Dec 1;25(6):e11-8.

12. Hatamleh R, Shaban IA, Homer C. Evaluating the experience of Jordanian women with maternity care services. Health Care Women Int. 2013 Jun 1;34(6):499-512. 
13. Bartsch E, Medcalf KE, Park AL, Ray JG. Clinical risk factors for pre-eclampsia determined in early pregnancy: systematic review and meta-analysis of large cohort studies. Bmj. 2016 Apr 19;353.

14. Townsend R, O'Brien P, Khalil A. Current best practice in the management of hypertensive disorders in pregnancy. Integr. Blood Press. Control. 2016;9:79.

15. Duley L, Henderson-Smart DJ, Walker GJ, Chou D. Magnesium sulphate versus diazepam for eclampsia. Cochrane Database Syst. Rev. 2010(12).

16. Chodzaza E, Bultemeier K. National management guidelines for obstetric Emergencies. AJM. 2010 Apr;4(2):57-61.

17. Browne JL, Van Nievelt SW, Srofenyoh EK, Grobbee DE, Klipstein-Grobusch K. Criteriabased audit of quality of care to women with severe pre-eclampsia and eclampsia in a referral hospital in Accra, Ghana. PLoS One. 2015 Apr 29;10(4):e0125749.

18. Kidanto HL, Mogren I, Massawe SN, Lindmark G, Nystrom L. Criteria-based audit on management of eclampsia patients at a tertiary hospital in Dar es Salaam, Tanzania. BMC Pregnancy Childbirth. 2009 Dec;9(1):1-9.
19. Muchiri D, Koigi-Kamau R, Kosgei RJ, Oyieke JB, Michoma P. Adherence to ministry of health guidelines in management of severe pre-eclampsia/ eclampsia in Pumwani maternity hospital, Kenya. East Afr. Med. J. 2016;93(6):232-7.

20. Tucker J, Farmer J, Stimpson P. Guidelines and management of mild hypertensive conditions in pregnancy in rural general practices in Scotland: issues of appropriateness and access. BMJ Qual. Saf. 2003 Aug 1;12(4):286-90.

21. Sibai BM. Etiology and management of postpartum hypertension-preeclampsia. AJOG. 2012 Jun 1;206(6):470-5.

22. Mone F, McAuliffe FM. Low-dose aspirin and calcium supplementation for the prevention of pre-eclampsia. Obstet. Gynaecol. 2014 Oct 1;16(4):245-50.

23. Oyston C, Baker PN. Therapeutic strategies for the prevention and treatment of pre-eclampsia and intrauterine growth restriction. Obstet. Gynaecol. Reprod. Med. 2013 Dec 1;23(12):375-80. 